\title{
Randomized controlled trial of intra-articular ketorolac on pain and inflammation after minor arthroscopic knee surgery
}

Nina Solheim, Lovisenberg Diakonal Hospital, Oslo, Norway

and Faculty of Medicine, Institute of Clinical Medicine, University of Oslo, Oslo, Norway

Ida Gregersen, Research Institute of Internal Medicine, Rikshospitalet, OUS, Oslo, and Faculty of Medicine, Institute of Clinical Medicine, University of Oslo, Oslo, Norway Bente Halvorsen, Research Institute of Internal Medicine, Rikshospitalet, OUS, Oslo and Faculty of Medicine, Institute of Clinical Medicine, University of Oslo, Oslo, Norway Vigdis Bjerkeli, Research Institute of Internal Medicine, Rikshospitalet, OUS, Oslo, and Faculty of Medicine, Institute of Clinical Medicine, University of Oslo, Oslo, Norway Audun Stubhaug, Department of Pain Medicine and Research, Division of Emergencies and Critical Care, Oslo University Hospital, Oslo, Norway and Faculty of Medicine, Institute of Clinical Medicine, University of Oslo, Oslo, Norway Torsten Gordh, Department of Surgical Sciences, Pain Medicine, Uppsala University, Uppsala, Sweden

Leiv A Rosseland, Department of Research and Development, Division of Emergencies and Critical Care, Oslo University Hospital, Oslo, Norway

and Faculty of Medicine, Institute of Clinical Medicine, University of Oslo, Oslo, Norway

Corresponding author:

Leiv Arne Rosseland, Division of Emergencies and Critical Care, Oslo University Hospital, Postbox 4950 Nydalen, 0424 Oslo, Norway

Word count: 3036

Short title: Local effects of ketorolac 


\section{Abstract}

\section{Background}

Ketorolac is an effective non-steroidal anti-inflammatory drug, commonly used with local anaesthetics as part of local infiltration analgesia protocols following orthopaedic surgery. However, systemic uptake and drug action may be the major mechanism after local infiltration. The aims of this project were to study the effects of a small, systemically ineffective dose of ketorolac given intra-articularly for postoperative pain and also to study synovial inflammatory biomarkers. We investigated whether ketorolac affects pro-inflammatory biomarkers in an in vitro model, as well.

\section{Methods}

In this placebo-controlled, blind, randomized study, we analysed intra-articular ketorolac $(5 \mathrm{mg})$ in ambulatory minor knee surgery patients with moderate or severe pain $(n=44)$. We assessed postoperative pain intensity $(n=44)$ and analysed microdialysis samples taken from knee synovial tissue every 20 minutes $(n=34)$. We also tested cyclooxygenase-independent effects of ketorolac in synovial cells stimulated by prostaglandin $\mathrm{E}_{2}$ and chondroitin sulphate in vitro.

\section{Results}

Intra-articular ketorolac (5mg) administration did not reduce pain or synovial pro-inflammatory cytokines CXCL1, IL-8, and MCP-1 0-120 minutes after knee arthroscopy. Female gender was a risk factor for moderate or severe pain (relative risk $1.45,95 \%$ confidence interval 1.04-2.01). Paradoxically, ketorolac increased the release of CXCL1 and IL-8 in prostaglandin $\mathrm{E}_{2}$ and chondroitin sulphate-stimulated synovial cells in vitro.

\section{Conclusion}


Ketorolac prescribed at a low dose intra-articularly does not produce any detectable analgesic effect after minor knee surgery. 
Local infiltration analgesia (LIA) via peri- or intra-articular injection has emerged as a common technique for acute pain control following orthopaedic surgery. Although local anaesthetics are always part of the drug combination, the adjuvants used in these protocols may vary. In a recent meta-analysis of LIA during knee surgery, the list of adjuvants included morphine, epinephrine, ketamine, glucocorticoids, and non-steroidal anti-inflammatory drugs (NSAIDs). ${ }^{1}$ The peripheral pharmacologic effects of the adjuvant drugs are insufficiently studied, and the systemic effects after local infiltration may be significant, but are not controlled for in most LIA studies. Most protocols which include local infiltration of NSAIDs have only studied doses of 15 to $60 \mathrm{mg}$ of ketorolac, generating high local drug concentrations. ${ }^{2}$

Acute pain intensity correlates with the concentration of inflammatory biomarkers, such as prostaglandin $\mathrm{E}_{2}\left(\mathrm{PGE}_{2}\right)$, pro-inflammatory cytokines, and chemokines. ${ }^{3}$ The pain-relevant biomarkers CXCL1, IL-8 (CXCL8), and MCP-1 are chemokines with pro-nociceptive effects and low molecular size allowing passage over the microdialysis membrane. ${ }^{4,5}$ The pharmacologic effect of NSAIDs is primarily to inhibit cyclooxygenase (COX) 1 and 2, with varying isoenzyme specificity. Furthermore, NSAIDs may have COX-independent relevant effects for inflammatory pain. ${ }^{6,7}$ Ketorolac is not well characterized in this regard.

Thus, the aims of this project were to study the local effect of ketorolac administered in the knee joint compared to placebo, analyse group differences in repeated assessments of pain intensity and local inflammatory mediators using synovial sampling (microdialysis) from the surgical site, and explore COX-independent effects of ketorolac on relevant biomarkers in $\mathrm{PGE}_{2^{-}}$and chondroitin sulphate-stimulated human synoviocytes in vitro. The expected finding was that intra-articular ketorolac produce pain relief and that 
anti-inflammatory effect is detectable in vivo and in vitro. The hypothesis in the randomized controlled trial (RCT) was that pain and inflammation were reduced after intra-articular injection of ketorolac. 


\section{Methods}

The Medical Ethics Committee for Health Region South East in Norway approved this study protocol, including biobanking of study samples (2009/1261a). The protocol conformed to the Declaration of Helsinki, and the study was conducted according to good clinical practice. The trial was registered in an international registry of interventional studies (clinicaltrials.gov identifier: NCT00774540) and was planned, conducted, and reported according to the CONSORT statement. ${ }^{8}$ All patients received oral and written information about the study before they signed the consent form. This was a parallel group (group sizes 1:1), placebo-controlled, randomized study.

\section{Patients}

We invited 80 adult (age $>18$ years) patients with American Society of Anaesthesiologists (ASA) physical status 1 and 2, who were scheduled for ambulatory surgery knee arthroscopic procedures at Lovisenberg Diakonal Hospital, to participate in this study if they were able to understand the information presented in Norwegian. The primary author (N.S.) recruited all patients during the pre-anaesthetic evaluation. Lovisenberg Diakonal Hospital is a large orthopaedic centre receiving patients representing the general Norwegian population. Exclusion criteria were as follows: known intolerance or contraindications to ketorolac or alcohol; history of drug abuse; pregnancy or breast-feeding; comprehensive arthrosis or synovitis; renal failure; heart failure with easily provoked symptoms; active gastric or duodenal ulcer, bleeding or perforation; increased bleeding tendency; liver cirrhosis; simultaneous participation in other trials; preoperative glucocorticoid; paracetamol in the last $12 \mathrm{~h}$; NSAIDs in the last 12-24 h; COX2 inhibitors in the last 24-48 $\mathrm{h}$; perioperative tourniquet; moderate to severe preoperative pain; or impending risk of medical drug interactions with ketorolac. In addition, based on previous studies in the same population, we recommended that 
patients withdraw their participation in the rare case of experiencing very severe pain immediately after surgery as such patients are better treated with iv opioids.

\section{Pain assessment}

Preoperatively, we instructed patients in the assessment of pain intensity on a 0-100 mm visual analogue scale VAS ( $0=$ no pain, 100=unbearable pain $)$ and also recorded preoperative pain intensity.

\section{Anaesthetic procedure}

If needed, midazolam (1-2 mg i.v.) was administered $1 \mathrm{~h}$ before induction. General anaesthesia consisted of propofol (target controlled infusion) and remifentanil (0.1-0.3 $\mu \mathrm{g} / \mathrm{kg} / \mathrm{min}$ i.v.). Airways were secured with a laryngeal mask and subsequently ventilated with $30-50 \%$ oxygen under continuous monitoring of vital parameters. Fentanyl $(1 \mu \mathrm{g} / \mathrm{kg}$ i.v. $)$ was eventually given to prevent remifentanil-induced postoperative hyperalgesia. No other analgesics were administered, thus eliminating confounding factors dependent on drug-induced pain relief. During surgery, a 20G intra-articular catheter was placed for drug administration. The method has previously been described in detail. $^{9}$

\section{Surgical procedure and microdialysis}

At the conclusion of the arthroscopic procedure, we implanted a microdialysis catheter (pore size, $20 \mathrm{kDa}$; CMA Microdialysis, Stockholm, Sweden) in the synovial membrane under arthroscopic visual control. For reference measurements, another microdialysis catheter was inserted subcutaneously in the abdomen. We used perfusion fluid T1 (CMA Microdialysis; $147 \mathrm{mmol} / \mathrm{L} \mathrm{Na}, 4 \mathrm{mmol} / \mathrm{L} \mathrm{K}, 2.3 \mathrm{mmol} / \mathrm{L} \mathrm{Ca} ; 156 \mathrm{mmol} / \mathrm{L} \mathrm{Cl}, \mathrm{pH}$ ), pumped through the 
outer tube using a CMA 106 pump (CMA Microdialysis), which flowed underneath the membrane, where the exchange between interstitial fluid and perfusion fluid occurs. Fluid flowed backward and microdialysis fluid collection took place via microvials exchanged every 20 minutes with perfusion speed was set at $2.0 \mu \mathrm{L} / \mathrm{min}$.

\section{Postoperative observations}

We requested that all patients verbally estimate their pain intensity as none, mild, moderate, severe, or very severe. Patients having very severe pain in the immediate postoperative period were advised to withdraw their participation and were offered i.v. alfentanil. Patients who reported moderate or severe pain between 0 and 120 minutes after the end of surgery were included in the randomized controlled trial and were asked to rate their pain intensity on a $0-100 \mathrm{~mm}$ VAS at baseline and every 20 minutes after inclusion in the study. Pain intensity was registered at rest. On the same time points the patients were ask to report side effect. The patients having only mild or no pain were not included in the RCT. All registration of patient reported outcome measures were done by the primary author.

\section{Randomization and intervention}

Eligible patients who reported moderate or severe pain were randomized to intra-articular injection of ketorolac $\left(5 \mathrm{mg}\right.$, Toradol ${ }^{\circledR} 10 \mu \mathrm{g} / \mathrm{mL}$, Roche, Basel, Switzerland) in $5 \mathrm{~mL}$ normal saline or intra-articular injection of $5 \mathrm{~mL}$ normal saline (placebo). The intra-articular catheter technique has been previously described. ${ }^{9}$ The randomization and test medicine production were performed by the pharmacy department at Lovisenberg Diakonal Hospital. A randomization list based on a random number generator was used and the block size was un-known to the investigators until 
all data were entered into the database and locked. Syringes with test medicine were labelled with general information about the study and patient number. The patients, all health professionals and researchers involved in the study, were blinded to the patient allocation.

\section{Microdialysis samples}

Samples for analysis of inflammatory biomarkers consisted of one microvial collected by continuous microdialysis from synovial tissue at the surgical site at 20-minute intervals. Control samples from a non-painful area were obtained from the microdialysis probe inserted in abdominal subcutaneous fat tissue as noted above. Samples were sent to a biobank, stored at $-70^{\circ} \mathrm{C}$ and then analysed after inclusion of the last study patient.

\section{Multiplex Proximity Extension Assay}

We analysed synovial microdialysis fluid using the Proximity Extension Assay (PEA), which allowed simultaneous measurement of 92 biomarkers with the requirements of only $1 \mu \mathrm{L}$ sample volume, ${ }^{10}$ and assessed using the Proseek ${ }^{\circledR}$ Multiplex Inflammation I panel (Olink Bioscience, Uppsala, Sweden) according to manufacturer's instructions. The protocol called for mixing of $3 \mu \mathrm{L}$ of incubation mix containing two antibodies labelled with single-stranded DNA-oligonucleotides d with $1 \mu \mathrm{L}$ serum and incubated at $8^{\circ} \mathrm{C}$ overnight; then, we added $96 \mu \mathrm{L}$ of extension solution containing PEA enzyme and PCR reagents to the mixture and incubated for five minutes at room temperature to allow DNA-reporter sequences to form by proximity ligation of the A-oligonucleotide and B-oligonucleotide. We then transferred samples to a thermal cycler for 17 cycles of DNA amplification performed using quantitative PCR technique. Using a 96.96 Dynamic Array IFC (Fluidigm, CA, USA), prepared and primed according to the 
manufacturer's instructions, we loaded unique primer pairs for each cytokine onto the left side of the 96.96 Dynamic Array IFC and the protein expression program run in the Fluidigm Biomark reader according to the instructions for Proseek ${ }^{\circledR}$.

Molecules $>10 \mathrm{kDa}$ will not pass through the membrane of $10 \mathrm{kDa}$ microdialysis catheters. The chosen biomarkers - CXCL1 (chemokine (C-X-C motif) ligand 1; molecular weight 7 kDa), IL-8 (interleukin-8; molecular weight $8 \mathrm{kDa}$ ); and MCP-1 (monocyte chemotactic protein-1; molecular weight $11 \mathrm{kDa}$ ) - are exchangeable and therefore suitable as repeated outcome measures.

\section{Cell culture}

We cultured human synovial cells (American Type Culture Collection, Rockville, MD, USA) in Leibowitz medium (Sigma Aldrich, St. Louis, MO, USA) supplemented with $10 \%$ foetal bovine serum (FBS). The day before the experiment, we trypsinized the synovial cells and seeded them in 12-well dishes at a density of 300,000 cells $/ \mathrm{mL}$. On the day of the experiment, we cultured the cells in OptiMem (Invitrogen, Life Technologies Corporation, Paisley, UK), and added $\mathrm{PGE}_{2}(1 \mu \mathrm{M}$; Cayman Chemical Company, Ann Arbor, MI, USA) as well as chondroitin sulfate (Sigma Aldrich) to the experimental cells, with or without ketorolac. After $20 \mathrm{~h}$, we harvested cell-free supernatants and cell pellets and stored at $-80^{\circ} \mathrm{C}$, always adding vehicle of the stimulus to controls and un-stimulated cells. We examined cell cultures for lactate dehydrogenase leakage using a cytotoxicity detection kit (Roche Applied Science, Indianapolis, IN, USA).

We measured chemokine levels (CXCL1, IL-8, and MCP-1) in synovial cell supernatants using multiplex suspension array technology (BioPlex, Bio-Rad, Hercules, CA, US). 


\section{Statistical analysis}

Baseline characteristics, demographic data, and data from the in vitro assay are presented as mean (SD) if normally distributed or median (range) if not normally distributed. We applied the Kolmogorov-Smirnov test to test for normality, and used a double-sided student's t-test to compare mean values for normally distributed data. For non-normally distributed data, we compared mean values using a non-parametric two-sided Mann-Whitney U-test; we used the Pearson chi-square test to analyse the frequency of baseline categorical variables between male and female subjects, such as pain response versus pain non-response. To test for group differences using the Linear Mixed Model in version 23 of SPSS ${ }^{\circledR}$ (Statistical Packages for the Social Sciences, Chicago, IL, and USA), we used the repeated measurements of pain intensity and data from each biomarker. The primary outcome measure was group differences in pain over time (the interaction between time and randomization group). $P<0.05$ was considered significant (type III test of fixed effects, F-test). We performed pairwise comparisons in the mixed model, including Bonferroni correction if F-tests were significant. Residuals were checked for normality and we observed that assumption was satisfied for all variables except IL-8 and MCP-1 concentrations. Intention to treat analysis was performed with all randomized patients included and incidence of side effects was tested with chi square analysis.

\section{Sample size calculation}

We estimated sample size based on data from a previous trial of identical design including only patients with moderate to severe pain. ${ }^{9}$ The mean pain intensity was 50 $(\mathrm{SD}=15)$. A sample size of 14 in each group would give $>80 \%$ power to detect a $33 \%$ reduction in pain (SamplePower version 3.0.1 Statistical Packages for the Social Sciences, Chicago, IL). To account for a possible uneven number of patients between 
treatment groups due to the gender-stratified randomization, non-normal distribution of outcome data, and possible dropouts, we included 40 patients (group size 20). 


\section{Results}

\section{Patient characteristics}

Inclusion period was from October 2008 to April 2015. After adjusting for ineligible patients, we observed 65 patients ( 28 females, 37 males) postoperatively, and included 44 in the RCT (Figure 1). Demographic data, preoperative pain, and distribution of surgical procedures between the randomization groups are presented in Table 1. The distribution between pain responders and pain non-responders corresponded to previous discoveries, indicating that an average of $30 \%$ of the population recover without need for analgesics. Eighty-two percent (23 of 28) of women and 57\% (21 of 37) of men reported moderate to severe postoperative pain with a need for active treatment $(P=0.03)$. Thus, female gender was a risk factor for early development of moderate to severe pain intensity with a relative risk (RR) of 1.45 (95\% confidence interval [CI] 1.04-2.01). Among pain responders, the VAS was $46.4 \mathrm{~mm}$ in female patients (95\% CI 39.4-53.3) and $39.7 \mathrm{~mm}(95 \%$ CI 30.9-48.4) in male patients, and the mean time for inclusion was 23 minutes for females (95\% CI 14-31) and 21 minutes for males (95\% CI 16-26). We excluded two patients a few minutes after inclusion: One due to very severe pain, and one restless patient mobilized early, who left hospital within the intended $2 \mathrm{~h}$ observation.

\section{Pain relief}

Pain relief was not different between the ketorolac and placebo groups (Figure 2, $P=0.23)$. The consumption of rescue analgesic drug given as i.v. Morphine was $3.8 \mathrm{mg}$ (SD 2.9) in ketorolac and 3.8 $\mathrm{mg}$ (SD 1.9) in placebo group $(P=0.58)$. There were no side effects reported in either group. 
Microdialysis, inflammation

The trajectories of synovial concentrations for CXCL1, IL-8, and MCP-1 did not differ (Figure 3). We analysed reference tissue in 37 random samples. The concentrations in reference tissue were below the lower detection limit for IL-8 in 14 of 37 samples and for CXCL1 in 28 of 37 samples. Thus, we based the comparison of synovial tissue and reference tissue on the concentration of MCP-1 (34 of 37 samples). On average, the ratio of reference tissue concentration to that of synovial membrane samples was 0.77 (SD $=0.34)$.

Inflammatory effects of ketorolac on synoviocytes in vitro

Synoviocytes were exposed to $\mathrm{PGE}_{2}$ and chondroitin sulphate, simulating the nociceptive microenvironment in the surgically traumatized and inflamed synovial joint with debris from cartilage. In unstimulated cells, PGE2 significantly enhanced the release of CXCL1 and IL-8 from the synoviocytes but did not alter MCP-1 levels. Chondroitin sulphate did not have any effect on these factors alone; however, addition of ketorolac increased the release of CXCL1 and IL-8 from PGE2- and chondroitin sulphate-stimulated cells. Ketorolac also enhanced the release of MCP-1 from unstimulated cells, but not from stimulated cells (Figure 4). 


\section{Discussion}

In the present study, local intra-articular injection of $5 \mathrm{mg}$ ketorolac in patients with moderate-to-severe pain did not produce pain relief in a two hour observation period. The dose was lower than often given in LIA, but the local concentration was probably high above the local concentration obtained after systemic administration. The lack of detectable pain-relieving effect was surprising; a fact that questions the feasibility of injecting ketorolac locally. In general, including only patients with a clinically significant level of pain increases the internal sensitivity of analgesic drug trials, as pain intensity is higher and variation is greatly reduced. ${ }^{11,12}$ Pain intensity decreased rapidly in this study, regardless of treatment allocation, which is in line with results of previous studies. ${ }^{9,13-15}$ We cannot, however, rule out potential peripheral analgesic effects of intra-articular ketorolac based on this single trial. The strength of this clinical acute pain assay is that since we include all patients with significant pain, there is increased potential to detect real life effects of anti-nociceptive agents. The small sample size is a limiting factor.

The analgesic efficacy of systemic ketorolac is well documented. ${ }^{16,17}$ Systemic administration is associated with common adverse events and the alternative to include ketorolac as a component of LIA was supposed to diminish this risk. ${ }^{1}$ Most trials that include local ketorolac have used higher doses $(15-60 \mathrm{mg}),{ }^{1}$ and systemic effects are probable with doses of $\geq 10 \mathrm{mg} .{ }^{18}$ Neither peripheral mechanisms of action nor significant risk reduction compared to systemic administration has been demonstrated. Recently, Kuchálik et al demonstrated that local infiltration of $30 \mathrm{mg}$ ketorolac together with local anaesthetics reduced the plasma concentration of IL-6 $4 \mathrm{~h}$ after total hip replacement. ${ }^{19}$ We believe that this reported effect on IL-6 expression may be a systemic effect rather than a local effect. The plasma concentration of ketorolac after local 
infiltration is comparable to concentrations after intramuscular injection. ${ }^{20}$ Physicians should individualize analgesic therapy, and patients at increased risk of NSAID-related adverse events should not undergo LIA with ketorolac or any other NSAID treatment. ${ }^{21}$ Systematic reviews have concluded that LIA produce effective pain relief after major knee surgery, ${ }^{22}$ but that future studies should control for systemic effects of NSAID to allow more definite conclusions on the analgesic efficacy of the technique. A recent meta-analysis concluded that pain after major knee surgery can be more effectively relieved with peripheral nerve blocks than LIA, and while injuries may occur after both techniques, incidence of falls were less frequent after LIA ${ }^{23}$ Moderate or severe postoperative pain was perceived by a significantly higher proportion of women than men ( $82 \%$ vs. $57 \%)$. Randomization stratified for gender resulted in balanced sex distribution, and the groups were comparable regarding other demographic and clinical characteristics. When conducting trials of acute pain, the highly significant difference between male and female participants must be taken into account. ${ }^{24}$ For instance, women report higher acute pain intensity after knee arthroscopic procedures, but levels of local pro-inflammatory biomarkers are higher in men. ${ }^{25}$ In the present study, local inflammatory mediators were not affected by ketorolac compared to placebo treatment. The pain-relevant biomarkers CXCL1, IL-8 (CXCL8), and MCP-1 are chemokines with pro-nociceptive effects. Their molecular weights are well below the size limit for microdialysis membrane transfer, and analyses indicated feasibility for microdialysis studies.

Neutrophil granulocytes participate in the early immune response to a noxious stimulus, especially in nerve lesions, potentially attracted by nerve growth factor (NGF), CXCL1, CXCL2, IL-8, and leukotriene-B4. ${ }^{26}$ Monocyte-derived macrophages express many pain mediators, including reactive oxygen species and COX-derived prostaglandins that 
directly sensitize primary afferents. ${ }^{27}$ Interactions between sensory neurons, immune cells, and glial cells are highly intertwined, mediated by pro-inflammatory cytokines, and under the influence of naturally occurring anti-inflammatory cytokines, such as IL-10, IL-4, IL-1 receptor antagonist (IL-1ra), and transforming growth factor- $\beta$ (TGF- $\beta) .{ }^{28}$ Joint pain may be mediated by CXCL1 or IL- $8 .{ }^{4}$

In an experimental setup mimicking the in vivo environment, we assessed the modulatory effects of ketorolac on PGE2- and chondroitin sulphate-incubated synovial cells. This assay demonstrated that the cellular inflammatory response includes a significant increase in CXCL1 and IL-8. Interestingly, the addition of ketorolac seemed to enhance the local inflammatory response without any dampening effects on CXCL1, IL-8 or MCP-1 levels, or pain intensity. Ketorolac increased inflammation and cartilage degradation in an experimental assay in rats, ${ }^{29}$ and chondrocyte cell death in vitro. ${ }^{30}$ Our observations (in vitro and in vivo) did not support COX-independent anti-inflammatory local effects of ketorolac on synovial cells, and probably local infiltration is of limited value.

In contrast, some studies of COX inhibitors indicate peripheral anti-nociceptive activity. Interestingly, parecoxib, a pro-drug that requires activation in the liver, has been reported to be effective in a rat model of osteoarthrosis. ${ }^{31}$ The observed effect could have been COX-independent direct effects of parecoxib, but systemic effects of the active metabolite valdecoxib are more likely, bringing into question the validity of the study design.

To the best of our knowledge, this is the first clinical trial using repeated measurements of synovial biomarkers via membrane microdialysis with simultaneous pain registration in combination with proteomics analyses. In addition to being a small RCT with a long recruitment period, our analysis of local effects of ketorolac has some limitations, the most important of which is limited number of biomarkers available for analysis. The microdialysis membrane allowed transmembrane transport of small molecules only, and many relevant 
cytokines are oversized. Twenty minutes of microdialysis generates only $20 \mu \mathrm{L}$ per sample. The volume limits the available laboratory analysis methods and comprehensive proteomic mapping is impossible. The Proximity Extension Assay allows analysis of 92 biomarkers in less than $10 \mu \mathrm{L}$, and this effective assay partly compensates for the microdialysis-related limitations in molecule size and small sample volume. The rapid decrease in pain intensity after inclusion limits the trial sensitivity. The acute pain course after minor surgery as knee arthroscopic procedures is not comparable to joint replacement procedures. Furthermore, we were not able to assess pain during mobilization due to the fact that movements could interfere with the microdialysis technique and the patients were not allowed to mobilise. In addition the observation period was short and we based the adverse events rates on the patients' report of suspected side effects only. The study was monitored internally and had only one visit from the Norwegian Medicines Agency. The protocol adhered to departmental GCP procedures when the study was planned (2008) but during these years the requirements have changed.

\section{Conclusions}

Ketorolac given at a low dose intra-articularly does not produce any detectable analgesic effect after minor knee surgery. We found no ketorolac-specific group differences over time in the expression of pro-inflammatory cytokines in synovial tissue. Paradoxically, ketorolac increased levels of pro-inflammatory biomarkers (i.e. CXCL1 and IL-8) in PGE2 and chondroitin sulphate-stimulated synovial cells.

\section{Acknowledgements}

The authors thank Marie Essermark, RN, at Uppsala University Hospital, Uppsala, Sweden, for technical assistance, and Ellen Lund Sagen, Institute of Internal Medical Research, University of Oslo, Norway, for assisting in the quantitative laboratory measurements. The study was funded by Lovisenberg, Hospital, Oslo University Hospital, University of Oslo, the 
Regional Health Authorities of South East Norway (Grant ID 2010109), and Uppsala Berzelii Technology Centre for Neurodiagnostics.

Conflicts of interest: The authors declare no conflicts of interest. 


\section{References}

1. Seangleulur A, Vanasbodeekul P, Prapaitrakool S, Worathongchai S, Anothaisintawee T, McEvoy M, Vendittoli PA, Attia J, Thakkinstian A. The efficacy of local infiltration analgesia in the early postoperative period after total knee arthroplasty: A systematic review and meta-analysis. Eur J Anaesthesiol 2016; 33: 816-31.

2. Stalman A, Tsai JA, Segerdahl M, Dungner E, Arner P, Fellander-Tsai L. Ketorolac but not morphine exerts inflammatory and metabolic effects in synovial membrane after knee arthroscopy: a double-blind randomized prospective study using the microdialysis technique. Reg Anesth Pain Med 2009; 34: 557-64.

3. Sommer C, White F. Cytokines, Chemokines, and Pain. In: Beaulieu P, Lussier D, Porreca F, Dickenson AH, eds. Pharmacology of Pain. 1 edn. Seattle: IASP Press, 2010: 279-302.

4. Wigerblad G, Bas DB, Fernades-Cerqueira C, Krishnamurthy A, Nandakumar KS, Rogoz K, Kato J, Sandor K, Su J, Jimenez-Andrade JM, Finn A, Bersellini Farinotti A, Amara K, Lundberg K, Holmdahl R, Jakobsson PJ, Malmstrom V, Catrina AI, Klareskog L, Svensson CI. Autoantibodies to citrullinated proteins induce joint pain independent of inflammation via a chemokine-dependent mechanism. Ann Rheum Dis 2016; 75: 730-8.

5. Geven E, Davidson EB, Vitters E, Sloetjes A, Walgreen B, Blom A, van Lent P. The role of S100A9 in pain response during experimentally induced acute synovitis. Osteoarthritis and Cartilage 2017; 25: S380.

6. Tegeder I, Pfeilschifter J, Geisslinger G. Cyclooxygenase-independent actions of cyclooxygenase inhibitors. FASEB J 2001; 15: 2057-72.

7. Calatayud S, Esplugues JV. Chemistry, Pharmacodynamics, and Pharmacokinetics of NSAIDs. NSAIDs and Aspirin. Springer, 2016: 3-16.

8. Schulz KF, Altman DG, Moher D. CONSORT 2010 statement: updated guidelines for reporting parallel group randomised trials. BMJ 2010; 340: c332.

9. Rosseland LA, Stubhaug A, Sandberg L, Breivik H. Intra-articular (IA) catheter administration of postoperative analgesics. A new trial design allows evaluation of baseline pain, demonstrates large variation in need of analgesics, and finds no analgesic effect of IA ketamine compared with IA saline. Pain 2003; 104: 25-34.

10. Lundberg M, Eriksson A, Tran B, Assarsson E, Fredriksson S. Homogeneous antibody-based proximity extension assays provide sensitive and specific detection of low-abundant proteins in human blood. Nucleic Acids Research 2011; 39: e102-e02.

11. Stubhaug A, Breivik H. Postoperative analgesic trials: some important issues. In: Baillières, ed. Clinical Anaesthesiology. London: Baillière Tindall, 1995: 555-84.

12. Cooper SA. Single dose analgesic studies: the upside and downside of assay sensitivity. In: Max MB, R. P, E.M. L, eds. Advances in pain research and therapy. New York: Raven Press, 1991: 117-23.

13. Rosseland LA, Helgesen KG, Breivik H, Stubhaug A. Moderate-to-severe pain after knee arthroscopy is relieved by intraarticular saline: A randomized controlled trial. Anesth Analg 2004; 98: 1546-51.

14. Rosseland LA, Stubhaug A, Grevbo F, Reikeras O, Breivik H. Effective pain relief from intra-articular saline with or without morphine $2 \mathrm{mg}$ in patients with moderate-to-severe pain after knee arthroscopy: a randomized, double-blind controlled clinical study. Acta Anaesthesiol Scand 2003; 47: 732-38.

15. Solheim N, Rosseland LA, Stubhaug A. Intraarticular morphine $5 \mathrm{mg}$ after knee arthroscopy does not produce significant pain relief when administered to patients with moderate to severe pain via intraarticular catheter. Reg Anesth Pain Med 2006; 31: 506-13. 
16. De Oliveira GSJ, Agarwal D, Benzon HT. Perioperative Single Dose Ketorolac to Prevent Postoperative Pain: A Meta-Analysis of Randomized Trials. Anesth Analg 2012; 114 : 424-33.

17. Cepeda MS, Carr DB, Miranda N, Diaz A, Silva C, Morales O. Comparison of morphine, ketorolac, and their combination for postoperative pain: results from a large, randomized, double-blind trial. Anesthesiology 2005; 103: 1225-32.

18. Smith LA, Carroll D, Edwards JE, Moore RA, McQuay HJ. Single-dose ketorolac and pethidine in acute postoperative pain: systematic review with meta-analysis. Br J Anaesth 2000; 84: 48-58.

19. Kuchálik J, Magnuson A, Tina E, Gupta A. Does local infiltration analgesia reduce peri-operative inflammation following total hip arthroplasty? A randomized, double-blind study. BMC Anesthesiol 2017; 17: 63.

20. Affas F, Eksborg S, Wretenberg P, Olofsson C, Stephanson N, Stiller CO. Plasma concentration of ketorolac after local infiltration analgesia in hip arthroplasty. Acta Anaesthesiol Scand 2014; 58: 1140-45.

21. Warth LC, Noiseux NO, Hogue MH, Klaassen AL, Liu SS, Callaghan JJ. Risk of Acute Kidney Injury After Primary and Revision Total Hip Arthroplasty and Total Knee

Arthroplasty Using a Multimodal Approach to Perioperative Pain Control Including Ketorolac and Celecoxib. J Arthroplasty 2016; 31: 253-55.

22. Andersen LO, Kehlet H. Analgesic efficacy of local infiltration analgesia in hip and knee arthroplasty: a systematic review. Br J Anaesth 2014; 113: 360-74.

23. Terkawi AS, Mavridis D, Sessler DI, Nunemaker MS, Doais KS, Terkawi RS, Terkawi YS, Petropoulou M, Nemergut EC. Pain Management Modalities after Total Knee Arthroplasty: A Network Meta-analysis of 170 Randomized Controlled Trials. Anesthesiology 2017; 126: 923-37.

24. Rosseland LA, Stubhaug A. Gender is a confounding factor in pain trials: women report more pain than men after arthroscopic surgery. Pain 2004; 112: 248-53.

25. Solheim N, Östlund S, Gordh T, Rosseland LA. Women report higher pain intensity at a lower level of inflammation after knee surgery compared with men. PAIN Reports 2017. 26. Wang XM, Hamza M, Wu TX, Dionne RA. Upregulation of IL-6, IL-8 and CCL2 gene expression after acute inflammation: Correlation to clinical pain. Pain 2009; 142: 275-83.

27. Nathan CF. Secretory products of macrophages. J Clin Invest 1987; 79: 319-26.

28. Scholz J, Woolf CJ. The neuropathic pain triad: neurons, immune cells and glia. Nat Neurosci 2007; 10: 1361-68.

29. Irwin MG, Cheung KMC, Nicholls JM, Thompson N. Intra-articular injection of ketorolac in the rat knee joint: effect on articular cartilage and synovium. Br J Anaesth 1998; 80: 837-39.

30. Abrams GD, Chang W, Dragoo JL. In Vitro Chondrotoxicity of Nonsteroidal Anti-inflammatory Drugs and Opioid Medications. Am J Sports Med 2017: 0363546517724423.

31. Jean YH, Wen ZH, Chang YC, Hsieh SP, Tang CC, Wang YH, Wong CS. Intra-articular injection of the cyclooxygenase-2 inhibitor parecoxib attenuates osteoarthritis progression in anterior cruciate ligament-transected knee in rats: role of excitatory amino acids.

Osteoarthritis Cartilage 2007; 15: 638-45. 


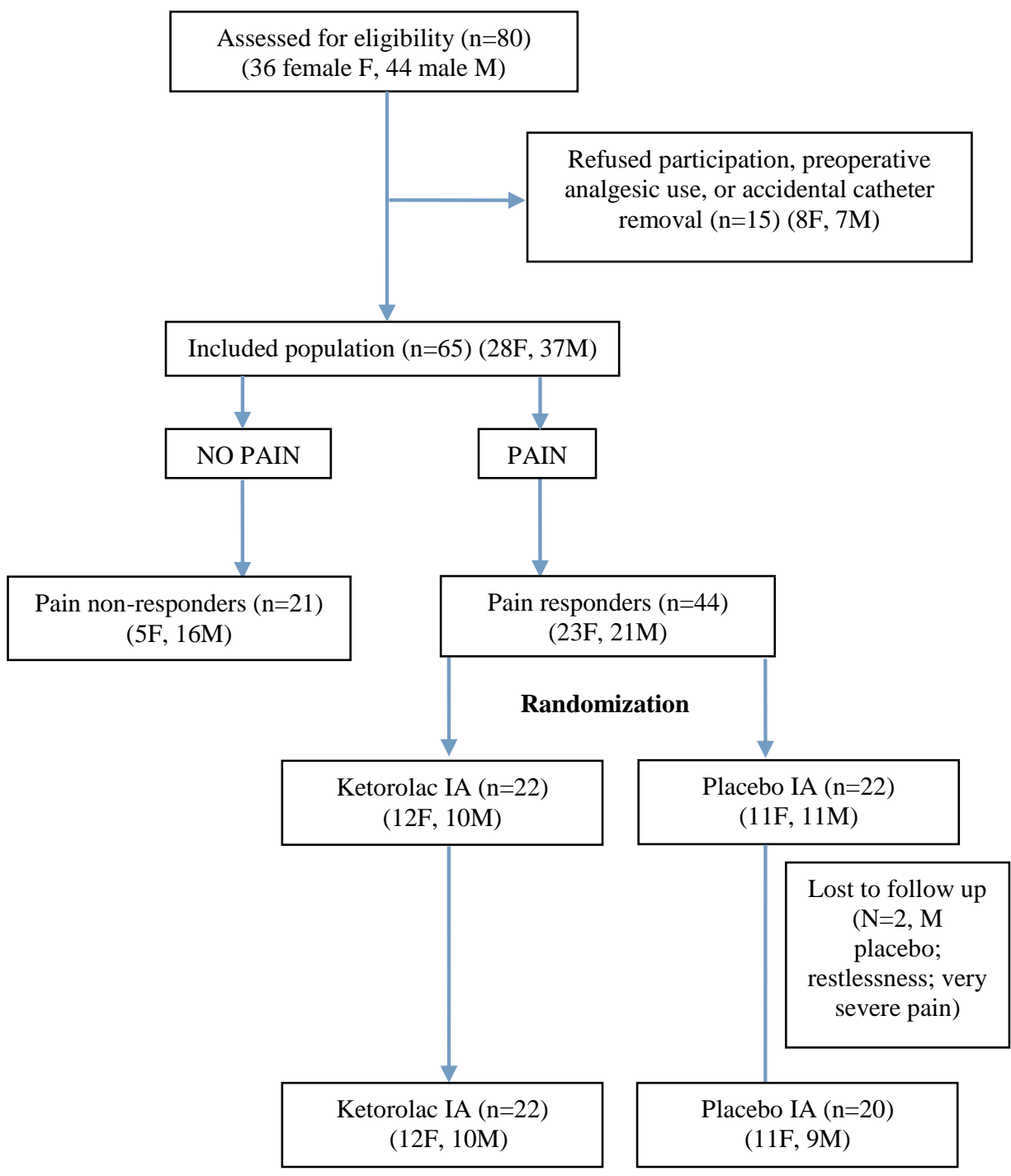

\section{Figure 1}

Flow chart of patient inclusion. F, females; M, males. 




Minutes from inclusion

Figure 2 Mean pain intensity based on a 0-100 mm visual analogue scale (VAS) for both groups. All patients reported moderate or severe pain postoperatively at inclusion. Error bars indicate standard deviation. The groups were not statistically significantly different. 
Figure 3
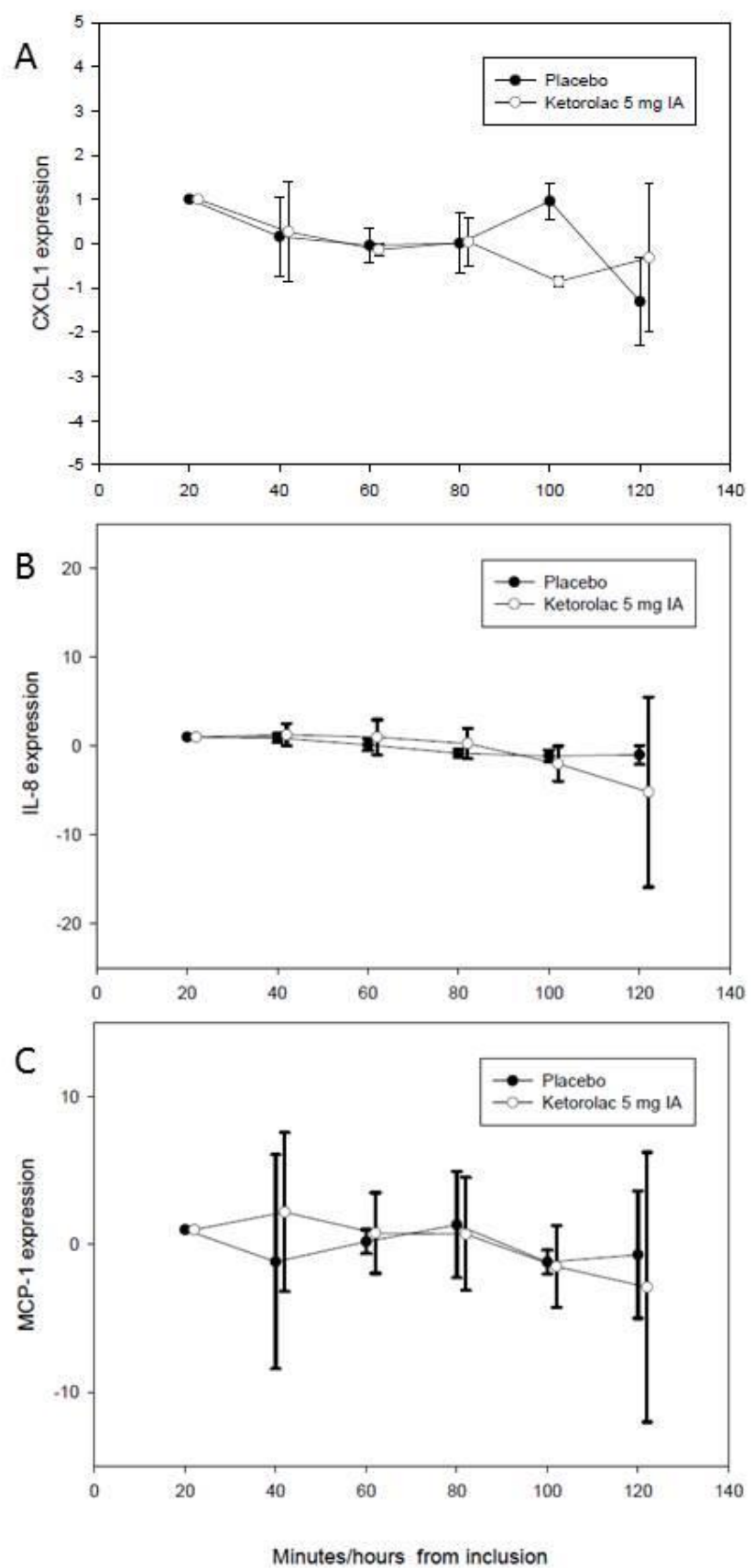

\section{Figure 3}

Mean protein expression in clinical intra-articular microdialysis samples. All included patients reported moderate or severe pain postoperatively at inclusion. Protein levels expressed as mean NPX-values are normalized to time 0 to show change from baseline. (A) CXCL1 (Gro $\alpha)$, (B) IL-8 (CXCL8), (C) MCP-1. Error bars indicate standard deviation. The groups were not statistically significantly different. 


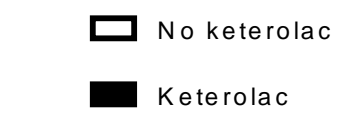

A

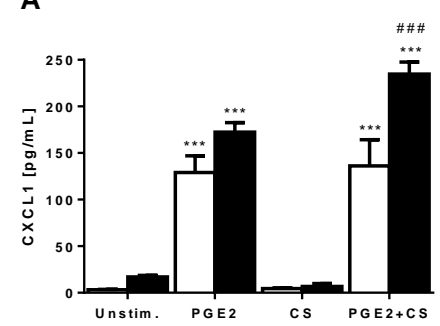

B

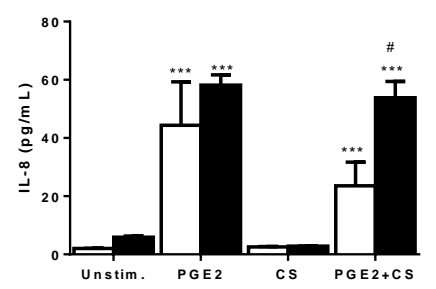

C

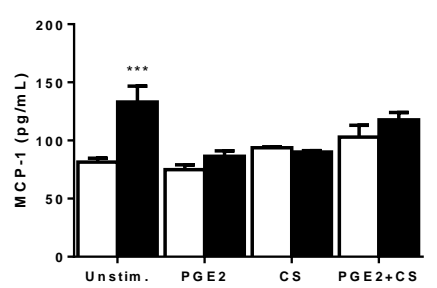

\section{Figure 4}

Inflammatory profile of synovial cells stimulated with PGE2 $(1 \mu \mathrm{M})$, chondroitin sulphate $(\mathrm{CS}, 5 \mu \mathrm{g} / \mathrm{ml})$, or a combination thereof in the absence or presence of ketorolac $(100 \mu \mathrm{g} / \mathrm{ml})$ for $20 \mathrm{~h}$. Medium was harvested and (A) CXCL1, (B) IL8, and (C) MCP-1 levels were assessed using suspension array technology. Data are presented as average \pm SEM. Statistical comparisons were performed using 2-way ANOVA. *** $P<0.001$ vs. unstimulated cells, $\# P<0.05$ and \#\#\# $P<0.001$ vs. cells in absence of ketorolac. 
Table 1

Demographics, preoperative pain, and surgical procedures in randomized patients $(\mathrm{N}=42)$

Ketorolac IA

$\operatorname{Sex}(\mathrm{M} / \mathrm{F})$

$10 / 12$

$43(12)$

Age (years)

Preoperative pain

intensity (0-100mm VAS)6.1 (9.0)

Surgical procedures:

Meniscectomies

Shaving

17 of 22

2 of 22

3 of 22
Placebo IA

$9 / 11$

$50(12)$

$11.7(11.4)$

17 of 20

0 of 20

3 of 20

The data are presented as number of patients or mean (SD). 